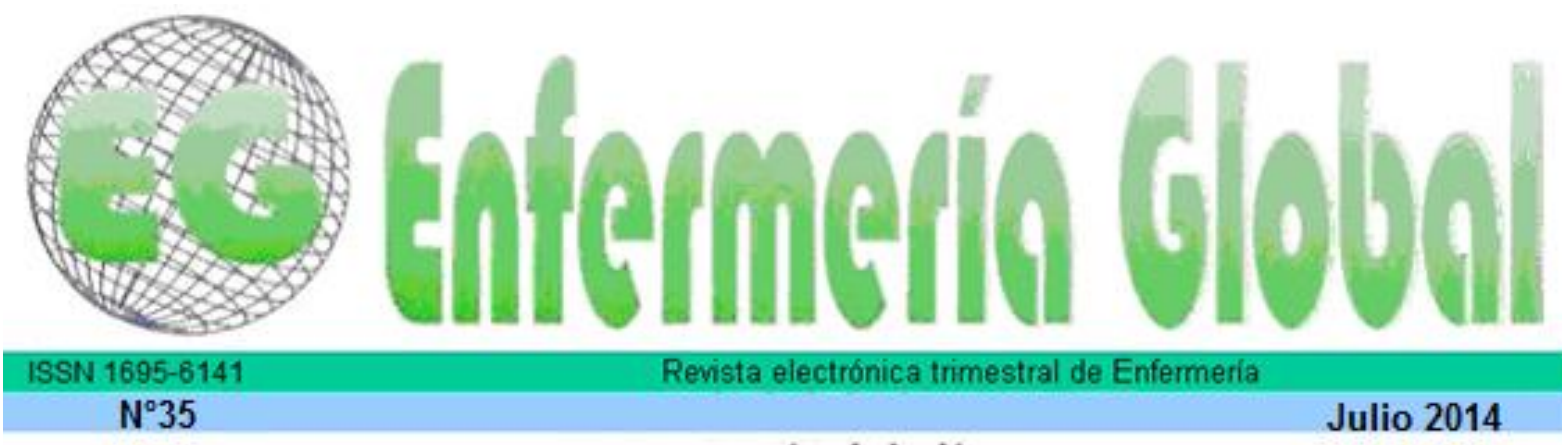

www.um.es/egloball

\title{
CLÍNIGA
}

\section{Heridas crónicas atendidas en un servicio de urgencias}

Chronic wounds treated in an emergency service of primary health care

\section{*Lorenzo Hernández, María Piedad **Hernández Cano, Rosa María ** Soria Suárez, María Isabel}

\begin{abstract}
*Enfermera. Servicio de Urgencias y Emergencias de Águilas. Murcia. E-mail:" azahara880@hotmail.com **Enfermera. UGC Cirugía General y Especialidades del HGB de Baza. Granada.
\end{abstract}

Palabras clave: Heridas crónicas; Urgencias; Atención Primaria; Atención Continuada; Cuidados de enfermería.

Keywords: Chronic wounds; Emergency; Primary Health Care; Continuing Care; Nursing care.

\section{RESUMEN}

Una herida crónica se define como una pérdida de continuidad de la superficie epitelial con pérdida de sustancia con escasa o nula tendencia a la curación espontánea y que requiere períodos muy prolongados para su cicatrización por segunda intención. Constituye un importante problema asistencial que afecta al sistema de salud por la disminución de la calidad de vida, su elevado coste económico y por la dedicación de mucho tiempo de los profesionales de Enfermería.

El objetivo de este estudio consiste en describir la prevalencia y tipo de heridas que padecen los pacientes atendidos en el Servicio de Urgencias, programados para fines de semana por su enfermero del centro de salud.

Los resultados obtenidos evidencian que casi la mitad de los pacientes acudían por presentar heridas crónicas, y que el $30 \%$ no traían indicaciones de su enfermero referente de la realización de la cura.

\section{ABSTRACT}

A chronic wound is defined as a loss of continuity of the epithelial surface with a loss of substance with little or no tendency to spontaneous healing and that requires very long periods for healing by secondary intention. It is an important health care problem affecting the health system because of the decreasing quality of life, their high cost and long-time dedication of professional nurses. 
The aim of this study is to describe the prevalence and the type of injuries suffered by patients treated in the Emergency Service, scheduled for weekends by his/her nurse at the out-patients clinic.

The results obtained show that almost a half of the patients attended the clinic for chronic wounds and the $30 \%$ of them did not bring their nurse indications concerning the realization of the cure.

\section{INTRODUCCIÓN}

Los pacientes que presentan Heridas Crónicas $(\mathrm{HC})$ acuden prácticamente a diario a su centro de salud para realizarse las curas o en el supuesto de padecer deterioro de la movilidad o inmovilización, es el personal de enfermería quien se desplaza al propio domicilio, siendo de gran importancia la labor de los servicios de urgencias que atienden a dichos pacientes en fines de semana y festivos, siguiendo una continuidad en los cuidados prestados.

Las HC constituyen uno de los más importantes problemas asistenciales que afectan al sistema de salud por la disminución de la calidad de vida de quien la padece y de sus cuidadores, su elevado coste económico y por la dedicación de mucho tiempo de los profesionales de enfermería. En España el coste anual de Tratamiento se ha estimado en torno a los 435 millones de euros correspondiendo el 18,9\% a Atención Primaria, el $28 \%$ a Atención Hospitalaria y el $53,1 \%$ a la Atención Sociosanitaria ${ }^{(1)}$. En el caso de las Úlceras de la extremidad inferior, según los estudios realizados en España, se pueden estipular los gastos totales de una visita, material, enfermería y cuidados médicos, que se cifran en 150 euros ${ }^{(2)}$; a los que habría que añadir el coste de la incapacidad laboral y tiempo empleado por sus familiares.

Una Herida Crónica es una herida que requiere para su cicatrización de períodos muy prolongados de tiempo, en 6 semanas no ha culminado el proceso de cierre de la misma, y que cicatriza por segunda intención, en un complejo proceso que elimina y reemplaza el tejido dañado ${ }^{(3)}$. No existe un consenso claro en la bibliografía revisada en cuanto a clasificación de HC como menciona Patricio Andrades y cols ${ }^{(4)}$, que afirma que existen multitud de clasificaciones de heridas, generalmente, dependientes del capricho del creador, considerando de gran relevancia determinar si la herida es aguda o crónica, basándose en conceptos de orden y temporalidad; de este modo considera como $\mathrm{HC}$ aquella que no sigue un proceso de reparación ordenado o que sigue un proceso de reparación que no restaura la integridad anatómica y funcional, estableciéndose unos 30 días para diferenciar una herida aguda de una crónica. En el Manual de prevención y cuidados locales de heridas crónicas ${ }^{(3)}$ elaborado por Cacicedo González y cols, reconocido de interés profesional por el GNEAUPP, dentro de las HC distinguen las Úlceras por Presión, Úlceras de Extremidad Inferior (arteriales, venosas y lesiones derivadas del pie diabético), y Úlceras Neoplásicas; así como algunas heridas agudas que pueden cronificarse por complicaciones como dehiscencia de suturas, heridas que fistulizan o bien heridas con evolución tórpida debido a infección de la misma; diferenciando las heridas crónicas de las heridas agudas, quirúrgicas o traumáticas, porque estas últimas cicatrizan por primera intención entre 7 y 14 días. Otros autores incluyen dentro de las $\mathrm{HC}$ el abdomen abierto entre otras ${ }^{(5)}$.

La curación de la herida se puede definir como un proceso que favorece la cicatrización del tejido cuya curación o remisión es el objetivo hasta el cierre completo de la misma ${ }^{(6)}$. En un proceso de cicatrización normal o curación por primera intención, se diferencian las fases de inflamación, proliferación y remodelación; en cambio, en las $\mathrm{HC}$, el proceso de cicatrización se realiza por segunda intención, 
prolongándose la fase de inflamación; en el que heridas con mayor profundidad, con mayor pérdida de tejido o que son consideradas como potencialmente infectadas y no son suturadas, dejando que la cicatrización evolucione en sus fases correspondientes, desde semanas a meses e incluso años, hasta su cierre espontáneo.

\section{Concepto y Tipos de Heridas Crónicas}

- Úlceras por presión. Lesión isquémica que se localiza en la piel y tejidos circundantes, producida por la combinación de factores extrínsecos como fuerzas de presión, cizallamiento y fricción, siendo determinante la relación presión/tiempo ${ }^{(3)}$. Aquí se incluyen las úlceras iatrogénicas, secundarias al tratamiento de un trastorno primario, producidas por mecanismos de roce y presión, como ocurre en la nariz por la exposición prolongada de mascarilla de oxígeno o sonda nasogástrica, en meato urinario a consecuencia del sondaje vesical prolongado, etc...

- Úlceras de la extremidad inferior. Se caracterizan por una pérdida de la integridad cutánea en la región anatómica comprendida entre la pierna y el pie ${ }^{(3)}$; producidas por una alteración de la circulación sanguínea venosa y arterial en las extremidades inferiores. Generan una gran demanda de asistencia sanitaria en todos los niveles, siendo imprescindible su tratamiento a nivel multidisciplinar. Se diferencian las úlceras venosas, arteriales y lesiones del pie diabético.

- Úlceras venosas. Se producen por una incompetencia funcional o anatómica de las válvulas produciéndose un reflujo sanguíneo que ocasiona una hipertensión venosa e insuficiente aporte de sangre a los tejidos provocando la aparición de necrosis y úlcera cutánea. Son las más frecuentes, aproximadamente un $80-90 \%$ son de esta etiología, afectando con más frecuencia a mujeres con edades comprendidas entre los 40 y 50 años ${ }^{(3)}$.

- Úlceras arteriales. Presentan una frecuencia de un $5 \%$ de las úlceras de la extremidad inferior, de aparición más frecuente en varones de 60 años aproximadamente. Es el resultado de una isquemia severa y prolongada en el tiempo (3). Tienen mal pronóstico, puesto que mientras no se restaure la circulación arterial no se podrá conseguir la curación.

- Pie diabético. La Sociedad Española de Angiología y Cirugía Vascular ${ }^{(7)}$, define el Pie Diabético como una alteración clínica, de base etiopatogénica neuropática, e inducida por la hiperglucemia mantenida, en la que con o sin coexistencia de isquemia, y previo desencadenante traumático, produce lesión y/o ulceración del pie. La úlcera de pie diabético no es una herida crónica por definición, sino que es una herida que se cronifica con el tiempo, bien por un retraso en el diagnóstico o por un tratamiento inadecuado de la misma; por lo tanto, nos encontramos con una lesión cuyo proceso de cicatrización está alterado.

- Úlceras neoplásicas. Son lesiones cutáneas que aumentan progresivamente de tamaño dando lugar a una úlcera abierta que no cicatriza y destruye el tejido circundante ${ }^{(3)}$. Algunos autores refieren que estas úlceras pueden aparecer en el $10 \%$ de pacientes con metástasis en la fase terminal de su enfermedad ${ }^{(8)}$. Generan un gran impacto tanto en el paciente y cuidadores por las repercusiones que conlleva la enfermedad así como en el personal de enfermería que atiende al paciente por las características de la lesión como el dolor, sangrado y mal olor. Se distinguen las úlceras neoplásicas originadas por el propio tumor, y secundarias a metástasis o por 
complicaciones de procedimientos terapéuticos como la quimioterapia por extravasación del citostático y la radioterapia.

\section{Factores de riesgo asociados}

La edad, fármacos consumidos por el paciente, tabaquismo, la malnutrición, obesidad, sedentarismo, situación sociofamiliar, patologías asociadas, tratamiento inadecuado de la herida son factores que van a repercutir negativamente en la cicatrización de la herida. Como factores de riesgo asociados se pueden mencionar, entre otros, la diabetes y otras enfermedades del riñón, hígado, pulmón, cáncer, un insuficiente aporte de oxígeno a los tejidos, sistema inmunológico debilitado o la presencia de una herida infectada o presencia de un cuerpo extraño.

\section{Abordaje de las Heridas Crónicas}

Para un correcto abordaje sanitario de las heridas crónicas, y mejora de la cicatrización de las mismas, en una valoración inicial se debe valorar y registrar la antigüedad de la lesión, localización, estadio en la que se encuentra, dimensiones, tunelización, estado de la piel perilesional, cantidad y tipo de exudado, signos de infección, presencia de dolor, olor, ... Tras la valoración, se debe planificar el tipo de cura a realizar indicando el tipo de desbridamiento realizado, cuidados locales y periodicidad de las curas. De suma importancia, al igual que la valoración, planificación de la cura, y correcto tratamiento es el registro del tratamiento que se le está realizando por el personal de enfermería para, de esta manera, asegurar una continuidad de los cuidados del paciente, en el caso de ser tratado por diversos enfermeros de la zona, y no interferir en la cicatrización de la herida, ya que en muchas ocasiones, cada enfermero usa tratamientos diferentes al iniciado, interfiriendo en el proceso de cicatrización.

El personal sanitario, generalmente, presta más atención a conseguir la cicatrización total de la herida, no teniendo en cuenta el dolor producido por la misma disminuyendo la calidad de vida de estos pacientes. Por ello, es importante realizar una valoración integral y centrarse no solo en la herida, sino también en las repercusiones que ésta tiene en el paciente y su calidad de vida.

\section{OBJETIVOS}

El objetivo del presente estudio fue evaluar la presencia de heridas crónicas en pacientes atendidos en el Servicio de Urgencias de Atención Primaria de ÁguilasMurcia, programados para fines de semana y festivos por su enfermero referente del centro de salud.

\section{METODOLOGÍA}

- Tipo de estudio: estudio descriptivo observacional, de corte transversal durante los meses de abril, mayo y junio del año 2012 (un domingo de cada uno) en el día que coincidía una de las autoras realizando su actividad laboral. Se realiza un análisis descriptivo mediante frecuencias y porcentajes. El análisis estadístico se realiza a través del software Microsoft Excel® 2003. 
- Población de estudio. Muestreo no probabilístico, obteniendo 40 pacientes atendidos en el Servicio de Urgencias para Enfermería Programada para curas y para Enfermería Programada a domicilio.

- Metodología. Se recogen los datos, a través de cuestionario heteroadministrado (cuestionario 1), a los pacientes que acuden al Servicio de Urgencias y los programados para acudir al domicilio, por la misma evaluadora evitándose la duplicidad de pacientes.

- Variables estudiadas. Se recogen las variables edad, sexo, tipo de herida, indicaciones por enfermero de atención primaria sobre el proceso de cura, tiempo de evolución (para excluir o incluir pacientes en estudio) y motivo que originó la cura.

- Ámbito del estudio. Estudio realizado en el Servicio de UrgenciasEmergencias (SUE) de Águilas, perteneciente a la Gerencia de Emergencias del 061 de Murcia.

- Criterios de inclusión. Se han recogido los datos a pacientes que presentan heridas crónicas, con una evolución de más de 4 semanas.

- Aspectos éticos. La participación se ha realizado tras informar previamente de los objetivos del estudio, dando los pacientes su consentimiento para su inclusión, asegurando el anonimato de los mismos.

\section{RESULTADOS}

Se atienden 94 pacientes para curas de heridas derivadas por su enfermero referente durante los días estudiados, donde 54 pacientes acudieron al servicio de urgencias para curas programadas, siendo excluidos del estudio por presentar heridas en proceso de cicatrización normal, relacionadas con quemaduras y suturas tras cirugía 0 incisiones accidentales. Por tanto, se escoge una muestra de estudio (n) de 40 pacientes que presentan heridas crónicas, de los cuales, el $95 \%{ }^{(38)}$ de los pacientes atendidos son mujeres y un $5 \%$ varones (2 pacientes), situándose la media de edad en 55, 9 años, desviación típica de 6,52 años, donde el menor cuenta con 42 años y el de mayor edad con 74 años.

De los pacientes tratados por enfermería por heridas crónicas (Gráfico1), en 25 casos $(62,5 \%)$ presentan heridas infectadas o de evolución tórpida, un 2,5\% (1 caso) para úlceras por presión. Acuden 14 pacientes (35\%) para curas de úlceras en extremidad inferior, correspondiendo 11 casos $(27,5 \%)$ a úlceras vasculares y venosas (no se diferencian entre ellas por falta de un diagnóstico de confirmación médico) y en 3 casos a heridas en pie diabético $(7,5 \%)$. 


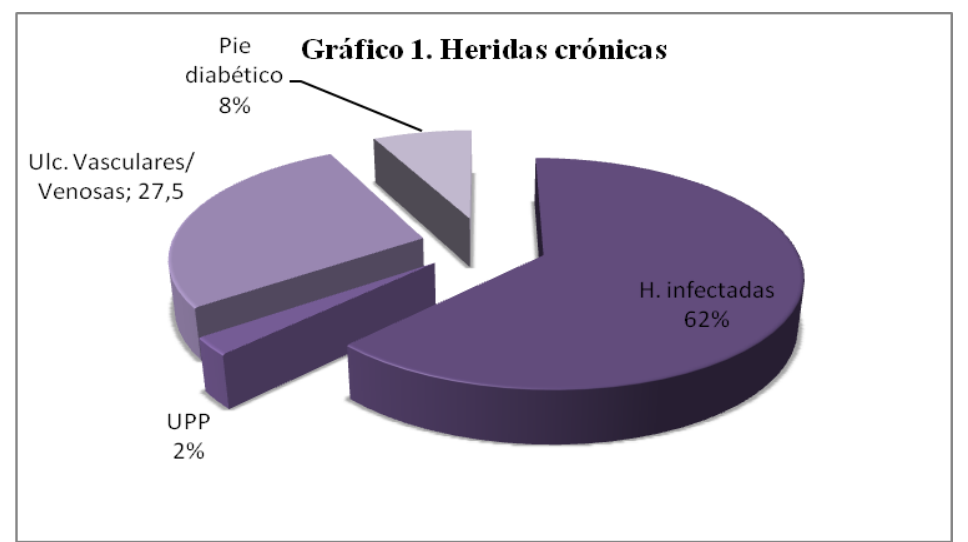

Respecto al lugar de asistencia por el personal de enfermería (Gráfico 2) se atienden 14 pacientes en su domicilio (35\%) y 26 pacientes (65\%) son atendidos en el servicio de urgencias tras acudir por medios propios.

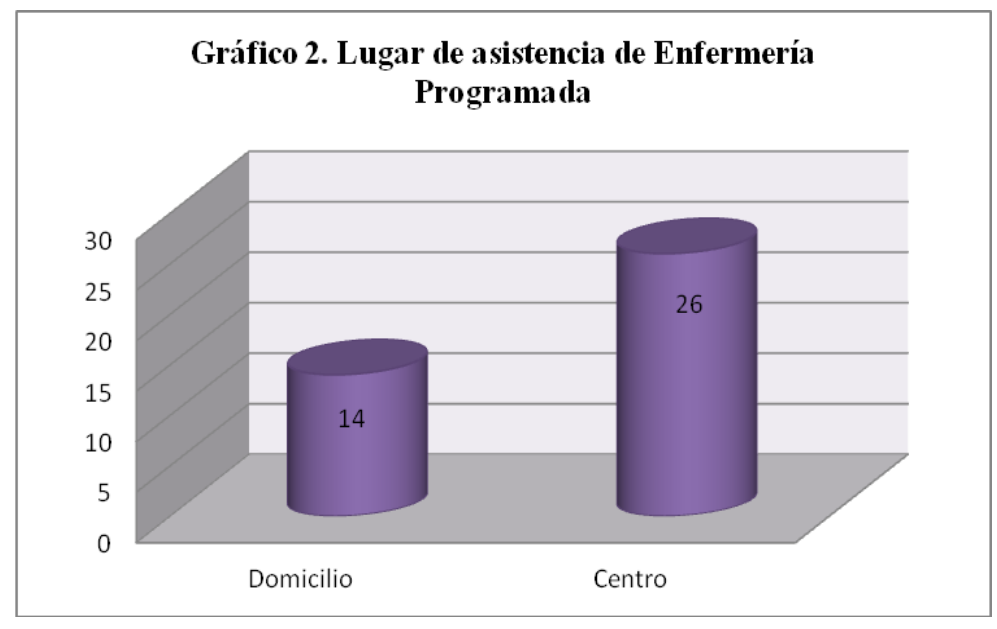

Sólo el 70\% (28) de los pacientes traían indicaciones de su enfermero de Centro de Salud de la pauta de realización de la cura, mientras que los 12 pacientes restantes no la traían $(30 \%)$ realizándose la cura a criterio del enfermero o según la indicación del paciente.

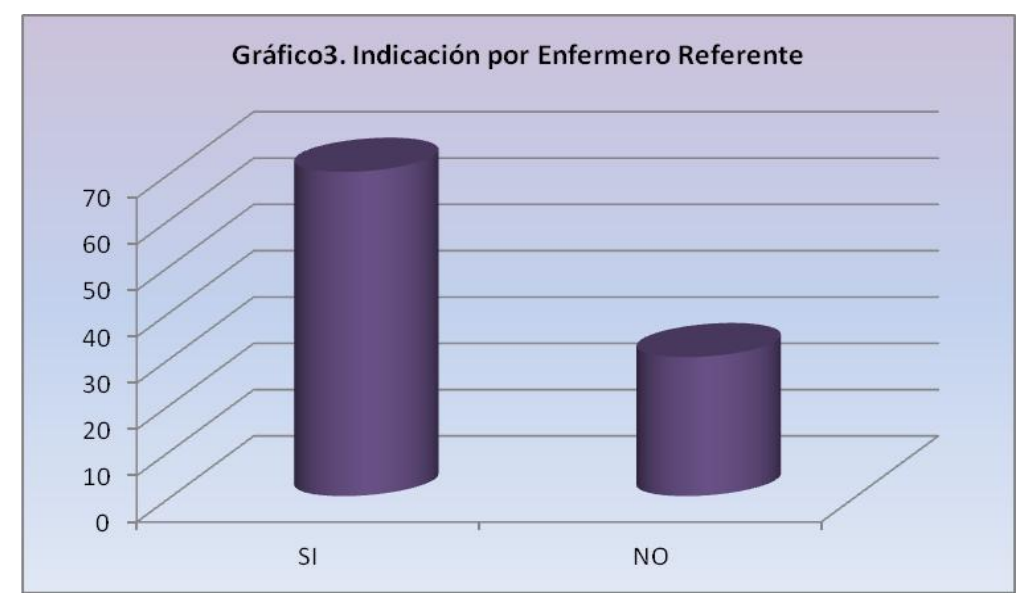

\section{DISCUSIÓN}

En nuestro estudio, la media de edad de los pacientes atendidos oscila en los 56 años, a diferencia del estudio de prevalencia por úlceras por presión realizado en 
España en 2005, donde refieren que el $84,7 \%$ de los pacientes atendidos por úlceras por presión pertenecen al grupo de edad de 65 años ${ }^{(9)}$; como ocurre en el estudio realizado en el Hospital General de Lanzarote, apareciendo mayor porcentaje de UPP en mayores de 60 años ${ }^{(10)}$ con un predominio del sexo femenino del 62,4\%. Según el estudio de prevalencia realizado en España por el Grupo Nacional para el Estudio y Asesoramiento en UPP (GNEAUPP) en 2006, se conoce que las mujeres son el sector mayormente afectado ${ }^{(11)}$, como ocurre en nuestro estudio correspondiendo al $95 \%$ de los pacientes atendidos. En el tercer estudio nacional realizado por GNEAUPP en 2009 esta prevalencia de UPP disminuye al 80,7\% ${ }^{(12)}$.

En el estudio de prevalencia realizado en una comarca de la provincia de Barcelona, refieren que la edad media fue de 77,5 años y el $67,9 \%$ eran mujeres (13), no coincidiendo con nuestros resultados en cuanto a la media de edad de los pacientes atendidos.

Las heridas crónicas más prevalentes en el Servicio de Urgencias y Emergencias de Águilas, son las heridas infectadas o con mala evolución, seguidas de las úlceras de la extremidad inferior, que comprenden las úlceras vasculares, las úlceras venosas y las úlceras del pie diabético. A diferencia del estudio de Barcelona, respecto a la etiología, en nuestro medio las más afectadas son las heridas de mala evolución o infectadas, seguidas de las úlceras de la extremidad inferior - vasculares y venosas$(27,5 \%)$, respecto al 40,6 \% que refieren en este estudio. Así mismo, en nuestro medio, las úlceras por presión, en el momento del estudio, tienen una prevalencia baja, en comparación con estudios como el de Barcelona que oscila al 38,2\% ${ }^{(12)}$.

\section{CONCLUSIONES}

Con este estudio hemos pretendido conocer la situación en nuestro medio laboral de la prevalencia de heridas crónicas, derivadas para su continuidad asistencial por el personal de enfermería de Atención Primaria, ya que se desconocían datos al respecto. La prevalencia de heridas crónicas encontrada en nuestra investigación varía con estudios publicados, debido a su realización en centros de diferentes características, no encontrándose estudios publicados en Servicios de Urgencias de Atención Primaria, considerándose unas cifras obtenidas que no posibilitan su comparación con las obtenidas en otros estudios. Así mismo, la disposición de recursos asistenciales en cada centro o zona geográfica es diferente, tratándose cada herida de forma diferente, ya de por sí, en un Servicio de Urgencias no se dispone del mismo material que en el Centro de Salud, realizándose una cura, en ocasiones, con una metodología y material diferente.

Actualmente se atienden a muchos pacientes derivados de atención primaria en domingos y festivos para curas programadas, destacando la dificultad para la continuidad asistencial ya que no todos los atendidos traen indicación de su enfermero de familia para la realización de la cura, existiendo una barrera en la continuidad de cuidados que puede empeorar la evolución de la herida. Para optimizar los recursos económicos y los tiempos empleados por el personal de enfermería, se deberían utilizar, entre los centros de salud de atención primaria y servicios de urgencias que realizan los cuidados durante fines de semana y festivos, Guías de Práctica Clínica para unificar criterios y conseguir una continuidad asistencial, disminuyendo la incidencia y prevalencia, considerados como importantes indicadores de calidad de los cuidados enfermeros; facilitando la indicación terapéutica en el cuidado de la cura, siendo primordial el hecho de que el enfermero 
referente del paciente le facilite un volante indicando el tratamiento realizado, en los centros que no dispongan de acceso informático a la historia de salud digital para su consulta.

De suma importancia para disminuir la incidencia de las Heridas Crónicas es actuar desde la prevención en todos los niveles asistenciales, constituyendo el método más eficiente para abordar el problema.

\section{BIBLIOGRAFIA}

(1) Posmett J, Soldevilla JJ, Torra JE. Epidemiología y Coste de las UPP en España. Estudio GNEAUPP 2005. Libro de Abstracs del VI Simposio Nacional de UPP. GNEAUPP. Zaragoza 2006.

(2) Marín Paredero V. Costes sociales y económicos de las úlceras de la extremidad inferior. La opinión de los expertos. Actualidad y controversia en la curación de heridas. Glosa, S.L., 2002.

(3) Cacicedo González R, Castañeda Robles C, Cossío Gómez F, Delgado Uría A, Fernández Saíz B, Gómez España MV, Gómez Fernández A. Manual de Prevención y Cuidados Locales de Heridas Crónicas. Servicio Cántabro de Salud, 1ํo edición, 2011 [acceso 12 Mayo 2013]. Disponible en: http://www.gneaupp.es/app/adm/documentosguias/archivos/40 pdf.pd

(4) Andrades P, Sepúlveda S, González J. Curación avanzada de heridas. Rev Chilena de Cirugía, 2004. [acceso 12 Mayo 2013]; 56 (4): 396-403. [acceso 12 Mayo 2013]. Disponible en:

http://www.revistacirugia.cl/PDF\%20Cirujanos\%202004.../Rev.Cir.4.04.(18).AV.pdf

(5) Jiménez Jiménez CE. Terapia de presión negativa: una nueva modalidad terapéutica en el manejo de heridas complejas, experiencia clínica con 87 casos y revisión de la literatura. Rev colomb. Cir., 2007 [acceso 12 Mayo 2013]; 22(4): 229224. Disponible en: http://www.scielo.org.co/scielo.php?pid=S201175822007000400004\&script=sci arttext\&tlng=es

(6) Consenso sobre cicatrización de heridas crónicas. Sociedad Argentina de Dermatología, 2008 [acceso 12 Mayo 2013]. Disponible en: http://www.sad.org.ar/file download/18/cicatrizacion.pdf

(7) Rueda J, Aragón FJ, Giralt E, Capillas R, Gago M, García F. Pie diabético. En: Soldevilla JJ, Torra JE (eds). Atención Integral de las Heridas Crónicas, $1^{\underline{a}}$ Ed. Madrid: SPA 2004; 331-358.

(8) Soldevilla J. J.Cuidados de las heridas neoplásicas y manejo del linfedema. En: Astudillo W. et al. Cuidados Paliativos en Enfermería. San Sebastián-España 2003. Sociedad Vasca Cuidados Paliativos 107-121.

(9) Soldevilla Agreda, J.; Torra i Bou, J.-E.; Verdú Soriano, J.; Martínez Cuervo, F.; López Casanova, P.; Rueda López, J.; Mayán Santos, J.M.: 2º Estudio Nacional de Prevalencia de Úlceras por Presión en España, 2005. Epidemiología y Variables definitorias de las lesiones y pacientes.

(10) Hernández Martínez FJ, Jiménez Díaz JF, Rodríguez de la Vera BC, Navarro García E, Navarro García R. Cuidado de Heridas (Interdepartamental). Rev Canarias Médica y Quirúrgica, 2011, [acceso 10 Mayo 2013]; 50-59. Disponible en: www.acceda.ulpg.es/bitstream/10553/5755/1/0514198 00024 0007.pdf

(11) Torra JE, Soldevilla J et al. Primer Estudio Nacional de Úlceras de pierna en España. Estudio GNEAUPPUIFC-Smith\&Nephew 2002-2003. Epidemiología de las úlceras venosas, arteriales, mixtas y de pie diabético. Gerokomos 2004; 15 (4): 23047. 
(12)Soldevilla Agreda JJ, Torra i Bou JE, Verdú Soriano J, López Casanova P. 3.er Estudio Nacional de Prevalencia de Úlceras por Presión en España, 2009: Epidemiología y variables definitorias de las lesiones y pacientes. Gerokomos, 2011 [16 marzo 2013]; 22(2): 77-90. Disponible en: http://scielo.isciii.es/scielo.php?script=sci_arttext\&pid=S1134-

928X2011000200005\&lng=es. http://dx.doi.org/10.4321/S1134-928X2011000200005 (13) Ferrer Solá, M; Chirveches Pérez, E; Molist Señé, G; Molas Puigvil, M; Besolí Codina, A; Jaumira Areñas, E; y cols. Prevalencia de las heridas crónicas en una comarca de la provincia de Barcelona. Enferm Clin., 2009; [acceso 20 Marzo 2013]; 19:4-10. Disponible en: http://www.elsevier.es/es/revistas/enfermeria-clinica35/prevalencia-heridas-cronicas-una-comarca-provincia-barcelona-13133272originales-2009

\section{ANEXO \\ Cuestionario 1}

CUESTIONARIO SOBRE HERIDAS ATENDIDAS POR EL PERSONAL DE ENFERMERIA DEL SERVICIO DE URGENCIAS

El objetivo del presente cuestionario es realizar un estudio para conocer la prevalencia sobre las heridas crónicas atendidas por el personal de enfermería del Servicio de Urgencias de Atención Primaria de Águilas (Murcia), atendidos durante domingos y festivos. Se garantiza el anonimato de los pacientes, dando su consentimiento para el estudio.

FECHA

NOMBRE YAPELLIDOS (solo iniciales)

EDAD SEXO

TIEMPO DE EVOLUCION DE LA HERIDA

TIPO DE HERIDA

DIAGNOSTICADA POR MEDICO DE FAMILIA. $\quad \mathrm{SI}() \quad \mathrm{NO}()$

TRAE INDICACIONES DE SU ENFERMERO TIPO DE CURA. SI ( ) NO ( )

CURA REALIZADA: EN SERVICIO DE URGENCIAS ( ) DOMICILIO ( )

LOCALIZACIÓN DE LA HERIDA 